3 Dean AS, Zignol M, Falzon D, et al. HIV and multidrug-resistant tuberculosis: overlapping epidemics. Eur Respir J 2014; 44: 251-254.

4 Jenkins HE, Plesca V, Ciobanu A, et al. Assessing spatial heterogeneity of multidrug-resistant tuberculosis in a high-burden country. Eur Respir J 2013; 42: 1291-1301.

5 van den Hof S, Tursynbayeva A, Abildaev T, et al. Converging risk factors but no association between HIV infection and multidrug-resistant tuberculosis in Kazakhstan. Int J Tuberc Lung Dis 2013; 17: 526-531.

6 Wells CD, Cegielski JP, Nelson LJ, et al. HIV infection and multidrug-resistant tuberculosis: the perfect storm. J Infect Dis 2007; 196: Suppl. 1, S86-S107.

7 Podlekareva DN, Panteleev AM, Grint D, et al. Short- and long-term mortality and causes of death in HIV/ tuberculosis patients in Europe. Eur Respir J 2014; 43: 166-177.

\title{
The role of therapeutic drug monitoring in individualised drug dosage and exposure measurement in tuberculosis and HIV co-infection
}

To the Editor:

We read with interest the paper by Esposito et al. [1] reporting a difficult-to-treat extensively drug-resistant tuberculosis (TB) case. TB treatment in HIV-positive individuals can also be particularly challenging. An HIV-positive 26-year-old male showing excessive weight loss (body mass index $17.5 \mathrm{~kg} \cdot \mathrm{m}^{-2}$ ) and clinical deterioration was admitted to the Beatrixoord Tuberculosis Centre (University Medical Center Groningen, Haren, the Netherlands) for the treatment of pulmonary TB. Mycobacterium tuberculosis isolated from sputum appeared susceptible to all first-line drugs tested. The patient received rifampicin (RIF) $(600 \mathrm{mg})$, isoniazid (300 mg), pyrazinamide (1500 mg) and ethambutol (1200 $\mathrm{mg})$ under directly observed therapy. He started with emtricitabine $(200 \mathrm{mg})$, tenofovir $(245 \mathrm{mg})$ and raltegravir $(800$ mg twice daily) 2 weeks later as combination antiretroviral therapy. Therapeutic drug monitoring (TDM) was performed to evaluate the extent of the effect of RIF on raltegravir. Multiple blood samples were drawn over a period of $12 \mathrm{~h}$ to evaluate RIF exposure. Although raltegravir concentrations were adequate, strikingly low RIF concentrations were measured [2]. Unfortunately, RIF concentrations were not measured in an earlier stage of the treatment. Newly obtained plasma samples confirmed the low RIF concentration. To detect a potential decreased absorption, the same dosage of RIF was administered intravenously, which resulted in an acceptable RIF exposure (fig. 1). Drug-drug interactions influencing the absorption of RIF were not expected based on the concomitantly administered medication. The patient had no gastro-intestinal complaints and the faeces showed no presence of Giardia lamblia, Entamoeba histolytica and Cryptosporidium spp. Between the start of the TB treatment and the TDM day he had gained $11.2 \mathrm{~kg}$. No obvious signs were present that could account for the remarkably low drug exposure after oral administration. However, despite the decreased bioavailability of RIF, the patient responded well to therapy. Because a decreased bioavailability was noticed in a late phase of treatment and considering the positive treatment outcome, it was decided not to change treatment. The patient has remained clinically well and free of relapse 2 years after completion of TB treatment.

Drug exposure of first-line anti-TB drugs has gained renewed interest after the hollow-fibre infection model showed that the effectiveness of these drugs is driven by the ratio of area under the curve (AUC) of concentration-time to minimum inhibitory concentration (MIC) [3]. In addition, evidence is accumulating that subtherapeutic concentrations may contribute to acquired drug resistance and treatment failure [4]. A reduction of anti-TB drug exposure, in particular RIF, in HIV patients was reported earlier [5], but not in all studies [6]. Enteropathy caused by parasitic infections or by HIV itself (enterocyte apoptosis) and diarrhoea can often explain the reduced drug absorption in HIV patients. In our patient, the AUC from time zero to $24 \mathrm{~h}$ after dosing (AUC0-24) was $2.43 \mathrm{mg} \cdot \mathrm{h} \cdot \mathrm{L}^{-1}$ after oral administration and $29.92 \mathrm{mg} \cdot \mathrm{h} \cdot \mathrm{L}^{-1}$ after i.v. administration, resulting in an estimated bioavailability of $8.12 \%$. For this specific case we speculate that the exposures of concomitantly administered anti-TB drugs were sufficient and 
contributed to the microbial killing and hence the favourable treatment outcome. Furthermore, an additional susceptibility test revealed that the MIC for RIF was $<0.0625 \mathrm{mg} \cdot \mathrm{L}^{-1}$, which translates to an AUC0-24/MIC ratio of 38.88. The hollow-fibre infection model showed that maximal microbial killing was achieved by a RIF daily AUC0-24/MIC ratio of 24.14 [3]. It would seem conceivable in the case of our patient that, despite the low RIF exposure after oral administration, a positive treatment outcome was achieved due to the remarkably low MIC for RIF.

Our case strengthens the suggestion that RIF absorption from the digestive tract may be severely impaired in TB and HIV co-infected patients. Especially in the continuation phase of TB treatment, variability in drug exposure may result in virtual monotherapy if the exposure of one of the two given drugs is very low.

Although the World Health Organization treatment guidelines for TB [7] make no mention of it, TDM has the potential to optimise treatment and to prevent extremely low anti-TB drug exposure in TB and HIV co-infected patients, as suggested earlier for multidrug-resistant (MDR)-TB treatment [8]. A recent consensus statement on MDR-TB treatment has already anticipated that TDM will be part of future guidelines [9]. In general, TDM may show its added value in patients who do not respond to treatment because of malabsorption, drug-drug interactions, increased drug metabolism, diabetes or TB infection in sanctuary sites. Early detection of low drug exposure may help to improve treatment response and prevent development of further drug resistance [8].

Our observation emphasises not only that drug concentrations should be measured but also that drug susceptibility should be evaluated by determining a MIC value. TDM and drug susceptibility testing should go hand in hand because, when combined, these tools help tailor the AUC/MIC ratio and thereby optimise therapy. This reduces the risk of treatment failure due to low drug exposure [4]. At present, TDM of anti-TB drugs can be advocated in case of toxicity or failing treatment response, but data from randomised clinical trials to support a routine TDM programme for all TB and HIV co-infected patients is lacking. As obtaining a full pharmacokinetic curve is not feasible in most routine settings, limited sampling strategies to estimate drug exposure in combination with dried blood spot analysis may provide an alternative [10]. In addition, the number of patients needed for screening may be reduced if it becomes clear which target populations are at increased risk of subtherapeutic exposure. Cost/benefit ratios for TDM might be variable across subpopulations. Whether low drug exposure is relatively common in patients with TB and HIV co-infection is currently unknown. Only an adequately designed study can help clarify a possible role for routine TDM for subpopulations of TB patients.

To date, only few laboratories offer TDM for anti-TB drugs. We encourage laboratories to embrace the opportunity to perform TDM for first-line anti-TB drugs as has been done in the past for drug susceptibility testing. The first steps have been made to develop an international quality assurance system for TDM to support laboratories in the effort to provide a high-quality service to their physicians and patients. Certified reference laboratories may provide the services for second-line TB drugs using dried blood spot analysis [8].

A rational introduction of TDM in national programmes will probably improve adequate therapeutic management and contribute to elimination of TB [11]. TDM may help to prevent the emergence of drug

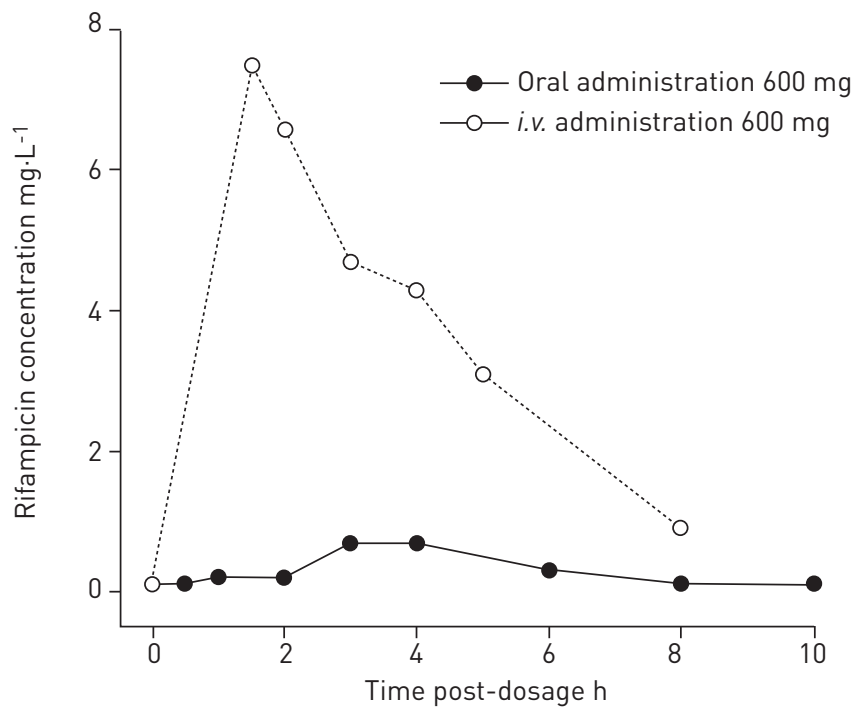

$\begin{array}{llr}\text { FIGURE 1. Serum rifampicin } \\ \text { concentrations } & \begin{array}{l}\text { over time, when } \\ \text { administered }\end{array} & \text { either orally or }\end{array}$


resistance and thereby the spread of MDR-TB. In addition, TDM may help to reduce the time to sputum conversion as it optimises drug exposure, thereby preventing transmission to others. For these reasons, TDM will probably be cost effective.

@ERSpublications

Wider use of TDM in complex TB cases could improve therapeutic management and prevent emergence of drug resistance http://ow.ly/CQx9j

Alper Daskapan $^{1}$, Wiel C.M. de Lange ${ }^{2}$, Onno W. Akkerman ${ }^{2}$, Jos G.W. Kosterink ${ }^{1,3}$, Tjip S. van der Werf ${ }^{2,4}$, Ymkje Stienstra $^{4}$ and Jan-Willem C. Alffenaar ${ }^{1}$

${ }^{1}$ University of Groningen, University Medical Center Groningen, Dept of Clinical Pharmacy and Pharmacology, Groningen, The Netherlands. ${ }^{2}$ University of Groningen, University Medical Center Groningen, Dept of Pulmonary Diseases and Tuberculosis, Groningen, The Netherlands. ${ }^{3}$ University of Groningen, Dept of Pharmacy, Section of Pharmacotherapy and Pharmaceutical Care, Groningen, The Netherlands. ${ }^{4}$ University of Groningen, University Medical Center Groningen, Dept of Internal Medicine - Infectious Diseases, Groningen, The Netherlands.

Correspondence: Jan-Willem C. Alffenaar, University of Groningen, University Medical Center Groningen, Dept of Clinical Pharmacy and Pharmacology, P.O. Box 30.001, 9700 RB Groningen, The Netherlands.

E-mail: j.w.c.alffenaar@umcg.nl

Received: Aug 042014 | Accepted after revision: Aug 162014

Conflict of interest: Disclosures can be found alongside the online version of this article at erj.ersjournals.com

\section{References}

1 Esposito S, D'Ambrosio L, Tadolini M, et al. ERS/WHO Tuberculosis Consilium assistance with extensively drug-resistant tuberculosis management in a child: case study of compassionate delamanid use. Eur Respir J 2014; 44: 811-815.

$2 \mathrm{Vu} \mathrm{DH}$, Koster RA, Wessels AM, et al. Troubleshooting carry-over of LC-MS/MS method for rifampicin, clarithromycin and metabolites in human plasma. J Chromatogr B Analyt Technol Biomed Life Sci 2013; 917-918: $1-4$.

3 Gumbo T, Louie A, Deziel MR, et al. Concentration-dependent Mycobacterium tuberculosis killing and prevention of resistance by rifampin. Antimicrob Agents Chemother 2007; 51: 3781-3788.

4 Srivastava S, Pasipanodya JG, Meek C, et al. Multidrug-resistant tuberculosis not due to noncompliance but to between-patient pharmacokinetic variability. J Infect Dis 2011; 204: 1951-1959.

5 Gurumurthy P, Ramachandran G, Hemanth Kumar AK, et al. Decreased bioavailability of rifampin and other antituberculosis drugs in patients with advanced human immunodeficiency virus disease. Antimicrob Agents Chemother 2004; 48: 4473-4475.

6 Conte JE, Golden JA, Kipps JE, et al. Effect of sex and AIDS status on the plasma and intrapulmonary pharmacokinetics of rifampicin. Clin Pharmacokinet 2004; 43: 395-404.

7 World Health Organization. Treatment of Tuberculosis: Guidelines. 4th Edn. WHO/HTM/TB/2009.420. Geneva, WHO, 2010.

8 Srivastava S, Peloquin CA, Sotgiu G, et al. Therapeutic drug management: is it the future of multidrug-resistant tuberculosis treatment? Eur Respir J 2013; 42: 1449-1453.

9 Lange C, Abubakar I, Alffenaar JW, et al. Management of patients with multidrug-resistant/extensively drug-resistant tuberculosis in Europe: a TBNET consensus statement. Eur Respir J 2014; 44: 23-63.

10 Vu DH, Alffenaar JW, Edelbroek PM, et al. Dried blood spots: a new tool for tuberculosis treatment optimization. Curr Pharm Des 2011; 17: 2931-2939.

11 D'Ambrosio L, Dara M, Tadolini M, et al. Tuberculosis elimination: theory and practice in Europe. Eur Respir J 2014; 43: 1410-1420.

Eur Respir J 2015; 45: 569-571 | DOI: 10.1183/09031936.00142614 | Copyright @eERS 2015

From the authors:

We read with interest the correspondence from A. Daskapan and colleagues commenting on our article [1] describing a complex tuberculosis (TB) outbreak in Milan, Italy, caused by extensively drug-resistant (XDR) strains of Mycobacterium tuberculosis [2,3].

In Rome, Italy, in July 2014, the European Respiratory Society (ERS) and the World Health Organization (WHO) developed the Global Framework for TB Elimination in countries with low TB incidence, which focused on the concept of TB elimination (defined as less than one TB case per million population) [4-7]. A recent ERS/WHO survey [7] demonstrated that several areas relevant for TB elimination are not fully 